\title{
Awake prone positioning in non-intubated patients with acute hypoxemic respiratory failure due to COVID-19: a systematic review and meta-analysis
}

\section{Ivan Pavlov}

CH Verdun: Centre Hospitalier de Verdun https://orcid.org/0000-0002-1805-8185

\section{Hangyong He}

Capital Medical University

\section{Bairbre McNicholas}

NUI Galway: National University of Ireland Galway

\section{Yonatan Perez}

CHRU Tours: Centre Hospitalier Regional Universitaire de Tours

\section{Elsa Tavernier}

CHRU Tours: Centre Hospitalier Regional Universitaire de Tours

Matthew W. Trump

The lowa Clinic

Julie A. Jackson

UnityPoint Health

\section{Wei Zhang}

Second Military Medical University First Hospital: Changhai Hospital

\section{Daniel S. Rubin}

UChicago Medicine

\section{Thomas Spiegel}

UChicago Medicine

\section{Anthony Hung}

UChicago Medicine

\section{Miguel Ángel Ibarra Estrada}

Civil Hospital of Guadalajara: Hospital Civil de Guadalajara

\section{Oriol Roca}

Vall d'Hebron Hospital: Vall d'Hebron Hospital Universitari

\section{David L. Vines}

Rush University

\section{David Cosgrave}

NUI Galway: National University of Ireland Galway

\section{Sara Mirza}

Rush University

John G. Laffey

NUI Galway: National University of Ireland Galway

\section{Todd W. Rice}

Vanderbilt University Medical Center

Stephan Ehrmann ( $\nabla$ stephanehrmann@gmail.com ) 


\section{Research}

Keywords: Coronavirus disease 2019 (COVID-19), Severe acute respiratory syndrome coronavirus 2 (SARS-Cov-2), Acute respiratory distress syndrome (ARDS), Acute hypoxemic respiratory failure (AHRF), Acute respiratory failure (ARF), Highflow nasal cannula (HFNC), Awake prone posi-tioning (APP), Non-invasive ventilation (NIV), Continuous positive airway pressure (CPAP)

Posted Date: January 26th, 2021

DOI: https://doi.org/10.21203/rs.3.rs-125426/v2

License: (c) (i) This work is licensed under a Creative Commons Attribution 4.0 International License. Read Full License 


\section{Abstract}

Background: Awake prone positioning (APP) has been advocated to improve oxygenation and prevent intubations of patients with acute hypoxemic respiratory failure due to coronavirus disease 2019 (COVID-19). This paper aims to synthesize the available evidence on the efficacy of APP.

Methods: We performed a systematic review and meta-analysis of observational studies to compare oxygenation parameters in-hospital intubation rate in patients treated with APP or with standard care.

Results: A total of 46 published and 4 unpublished observational studies that included 2994 patients were included. APP was associated with significant improvement of various oxygenation parameters in 19 studies $(n=381)$ that reported this outcome. The intubation rate was $27 \%(95 \% \mathrm{Cl}, 19$ to $37 \%)$ in the 870 patients treated with APP, as compared to $30 \%$ $(95 \% \mathrm{Cl}, 20$ to $42 \%)$ in the 852 patients treated with usual care $(p=0.71)$.

Conclusions: On the basis of the available evidence, it is not possible to demonstrate efficacy of APP for patients with COVID-19 acute respiratory failure, as assessed by the need for invasive ventilation. Routine implementation of APP outside of a clinical trial is not supported by current evidence. Randomized controlled clinical studies are urgently needed to definitively assess the utility of APP in these patients.

Registered on PROSPERO on August 3d, 2020, CRD42020201947.

\section{Introduction}

The coronavirus disease 2019 (COVID-19) pandemic has led to a sudden surge of hospital admissions for acute hypoxemic respiratory failure (AHRF). A significant proportion of patients who are hospitalized for COVID-19 fulfill the criteria for the acute respiratory distress syndrome (ARDS)[1], and require prolonged mechanical ventilation.

In the United Kingdom, among patients who were admitted to the intensive care unit (ICU), 72.1\% received advanced respiratory support, defined as mechanical ventilation (MV), or extracorporeal membrane oxygenation (ECMO), or endotracheal intubation[2], during their stay, with a median duration of MV of 13 days, and mortality rate of $48 \%$. Similar outcomes were reported elsewhere[3-5].

This surge of severely ill patients, who required high levels of care for protracted periods of time, threatened to overwhelm healthcare systems worldwide, especially in those regions with lower ICU capacity[6]. It is in this unusual setting in which both ICU beds and ventilators were in short supply, that clinicians turned to awake prone positioning (APP) as a means of avoiding - or, at least, delaying - endotracheal intubation, and thus lessening the pressure on the overburdened ICUs[714].

Prone positioning is one of the few interventions that has been proven to reduce mortality in intubated and mechanically ventilated patients with moderate to severe ARDS[15,16]. This effect is likely mediated through a combination of better lung recruitment, reduced ventilation/perfusion mismatch, and prevention of alveolar strain and ventilator-induced lung injury by a more homogenous distribution of pleural pressures throughout the lung parenchyma[17].

Prior to the pandemic, two small studies (combined $n=35$ ) explored the feasibility of APP of spontaneously breathing nonintubated patients[18,19]. In one study, eleven of 20 patients with moderate to severe ARDS were able to avoid intubation with the use of APP combined with either high-flow nasal cannula (HFNC) or non-invasive ventilation (NIV)[19]. Both studies demonstrated improvement of oxygenation, as measured by the ratio of arterial oxygen partial pressure $\left(\mathrm{PaO}_{2}\right.$ in $\mathrm{mmHg}$ ) to the fraction of inspired oxygen $\left(\mathrm{FiO}_{2}\right)[18,19]$. 
The first report of APP in patients with COVID-19 came from a Chinese retrospective study of 631 patients (10\% of them critically ill). A multi-pronged intervention that included early and aggressive use of HFNC combined with APP resulted in lower overall mortality (3.33\%, as compared to $4.34 \%$ in a nearby province). A very low percentage of patients required mechanical ventilation ( $<1 \%)$, as compared to the national average of $2.3 \%[7]$.

Since then, groups worldwide have reported on the use of APP in AHRF due to COVID-19, and showed improvement of oxygenation, and reduction of respiratory rate in populations with various disease severity[8-14]. However, whether improvement of surrogate physiological endpoints translates into better clinical outcomes, such as reduced incidence of intubation, or reduced mortality, remains unknown. Moreover, APP was variously combined with either conventional oxygen therapy, HFNC, or NIV, and for various periods of time. It is presently unknown whether the type of respiratory support impacts on the effects of APP.

The aim of this systematic review and meta-analysis was to investigate the hypothesis that APP of non-intubated patients with AHRF due to COVID-19 led sustained improvement of oxygenation, resulting in reduced intubation rate. The impact of APP on mortality, and the tolerability of APP are reported as exploratory secondary outcomes. We also explored the impacts of the duration of APP, the severity of the AHRF, the type of respiratory support (conventional oxygen therapy, HFNC, or NIV) on respiratory parameters, intubation rate, and mortality.

\section{Methods}

This study was registered on PROSPERO (CRD42020201947), and the detailed protocol is available at [https://www.crd.york.ac.uk/prospero/display_record.php?RecordID=201947]. Our findings are presented in conformity with the PRISMA guidelines[20].

\section{Search strategy and study selection}

Two investigators ( $\mathrm{HH}$ and JL) searched the MEDLINE, EMBASE, and PubMed, Web of Science, Scopus, MedRixv, BioRixv, ClinicalTrials.gov, and Wanfang databases for studies published from January 1st 2020 to August 15th 2020, with restrictions to English and Chinese languages. The keywords of ("prone position*" OR "Pron*") AND ("COVID-19" OR "SARS" OR "coronavirus") AND ("awake" OR "non-intubated" OR "conscious") were utilized to search literature evaluating APP for patients with COVID-19. This enabled the identification of cohorts of patients treated with APP. The keywords of ("nasal high-flow" OR "HFNC" OR "high-flow nasal cannula" OR "noninvasive ventilation" OR "NIV" OR "continuous positive airway pressure" OR "CPAP") AND ("COVID-19" OR "SARS" OR "coronavirus") were used to identify reports of patients treated with either HFNC or NIV/CPAP, without the use of APP, to be included as a control cohort. Equivalent keywords were used for searches in Chinese. Searches were supplemented with examination of reference lists in identified studies, and verbal communication with experts.

Studies were included if they met the following criteria: (1) original research reports of COVID-19 patients, (2) patients were treated with APP and/or HFNC/CPAP or NIV or conventional oxygen therapy. The exclusion criteria were: (1) languages other than English or Chinese, (2) study protocols, review articles, abstracts, editorials, (3) research on newborns or animals, (4) reports of fewer than 3 cases.

The investigators then independently parsed through the titles and abstracts of all identified articles and produced a list of potentially relevant papers. The full texts of these papers were then reviewed, and a final list of studies to be included in the meta-analysis was produced. Any disagreements were resolved by consensus.

Data from the articles were extracted by two independent teams ( $\mathrm{HH}$ and $\mathrm{JL}, \mathrm{BM}$ and YP) using a standardized data extraction form. Extracted data included the authors, year of study, country, patient characteristics, the type of respiratory 
support, the details of APP intervention, tolerability and outcomes. Any disagreements were resolved by consensus in the presence of all four investigators.

If the outcomes of intubation rate and mortality were not reported, or if it was not clear whether the patients received APP and for what duration, the corresponding authors were contacted for clarifications.

To enlarge the sample size, unpublished data provided by the investigators' institutions (BM, JJ, WZ, DR) was also included in the meta-analysis. Ethical approval was obtained at each institution prior to data collection.

\section{Pre-planned statistical analyses}

The main outcomes are and the proportion of physiological "responders" to APP and the in-hospital intubation rate. In conformity with established custom in the ARDS literature, responders were defined by an increase of $\mathrm{PaO}_{2} / \mathrm{FiO}_{2}$ ratio $\geq$ $20 \%[21]$. When the $\mathrm{PaO}_{2} / \mathrm{FiO}_{2}$ was not reported, an increase of $\mathrm{SpO}_{2} / \mathrm{FiO}_{2}$ ratio $\geq 20 \%$ was considered as a response, given the linear relationship between the two ratios[22,23]. In-hospital mortality rate is a complex outcome that is modulated by multiple individual and population-level confounders, and is the reported as an exploratory secondary outcome.

For dichotomous outcomes, we pooled proportions using a logit transformation with 95\% confidence intervals $(\mathrm{Cl})$. We assessed statistical heterogeneity by visual inspection of the forest plots and by calculating the $\mathrm{Q}$ and $\mathrm{I}^{2}$ statistics, which were interpreted according to conventional thresholds. For all analyses, we implemented random-effects models with inverse variance weighting, providing that at least three studies were available.

Potential sources of heterogeneity or inconsistency include baseline disease severity in terms of $\mathrm{PaO}_{2} / \mathrm{FiO}$ at the initiation of therapy, duration of APP, the timing of APP initiation, and the type of respiratory support (conventional oxygen therapy, HFNC, NIV). We investigated the distributions of these characteristics across groups and studies.

We pre-specified 3 characteristics in the protocol to be subject to subgroup analyses on the probability of intubation and mortality. When the information was available we restrained the analysis to the studies with $\mathrm{PaO}_{2} / \mathrm{FiO}_{2}<150 \mathrm{mmHg}$ vs. $\geq$ $150 \mathrm{mmHg}$ and according to respiratory support devices (HFNC vs. CPAP/NIV). The cut-off value of $\mathrm{PaO}_{2} / \mathrm{FiO}_{2}<150$ $\mathrm{mmHg}$ was based on the previously described signal of survival benefit when these patients are managed with early intubation, as compared to a non-invasive strategy[24]. The third subgroup analysis was restrained to studies in the group of APP, in which we analyzed the relationship between APP duration and the probability of intubation and mortality. For multiple comparisons, the Bonferroni correction was used to establish the threshold of statistical significance, thus all $p$ values were compared to the threshold of 0.0125 . We also performed a sensitivity analysis by excluding all data obtained from unpublished sources.

We did not formally assess bias of included studies, as all of them were observational, and inherently highly biased. We did not produce a funnel plot, as this method is inaccurate for meta-analyses of proportion studies[25].

\section{Post hoc comparator groups}

While collecting data, and before carrying out any analyses, we realized that only a minority of identified papers reported on "pure" populations in which either all patients were subjected to APP, or none were. We therefore decided to group patients into three groups a priori: (1) "APP" when all patients were proned, (2) "some APP" when some (at least 10\%) but not all patients were proned, and (3) "no APP" when no patients were proned (less than 10\%). Papers that focused on APP were classified as APP, regardless of the number or proportion of patients that were able to remain in PP. We compared 
patients treated with APP (group 1) with those not treated with APP (group 3), and we reported the p-value associated with the test for subgroup differences between group 1 and group 3. We also performed sensitivity analyses including group 2 as a separate subgroup in the analyses. While some patients were treated with conventional oxygen therapy in the "APP" group, only HFNC, CPAP and NIV were used in the "no APP". We therefore performed a sensitivity analysis by excluding patients treated with conventional oxygen therapy. We elected not to exclude these patients from our primary analysis, as the use of HFNC and CPAP or NIV was often prohibited early in the pandemic due to disease transmission considerations, and patients treated with conventional oxygen only were not necessarily less sick than those treated with other modalities. Finally, in order to test for geographical variation of care, we performed sensitivity analyses with restriction to high- or lowGDP countries.

All analyses were performed in R version 3.6.3[26], with the help of meta package[27].

\section{Results}

Our search strategy identified 173 publications on the subject of APP (Figure E1 in the online supplement), and 271 papers on the subject of non-invasive oxygenation modalities (Figure E2 in the online supplement) in severe COVID-19. Thus, a total of 444 potentially relevant publications were identified, and 440 were screened for inclusion after removal of duplicates (Figure 1). After full-text review, 46 published studies[8-10,12,14,28-68] and data from 4 unpublished datasets were included in the final review, with a combined 2994 subjects: 921 patients treated with APP, 870 patients treated without APP, and a group of 1203 patients in whom a significant proportion were treated with APP (Figure 1, Table 1, and Tables E1 and E2 in the online supplement). Clarifications and supplemental data were obtained from 18 corresponding authors.

\section{Physiological response to awake prone positioning.}

Nineteen studies $(n=381)$ reported on the physiological responses to APP. APP resulted in improved $\mathrm{SpO}_{2}$ or $\mathrm{PaO}_{2}$ in all 13 studies $(n=271)$ that reported on changes in oxygenation[8-10,28,30,33-35,40-43,45].

Ten studies $(n=198)$ reported on changes in the $\mathrm{PaO}_{2} / \mathrm{FiO}_{2}$ ratio[10,12,14,29,37,38,42,43,45,46], of them nine $(n=192)$ reported significant improvement in $\mathrm{PaO}_{2} / \mathrm{FiO}_{2}$ ratios with $\mathrm{APP}[10,12,14,37,38,42,43,45,46]$. Mean improvement was greater than our predefined threshold of $\geq 20 \%$ in all seven studies in which changes of $\mathrm{PaO}_{2} / \mathrm{FiO}_{2}$ ratios were reported in sufficient detail[14,37,38,42,43,45,46]. In three studies $(\mathrm{n}=72)$, the improvement of the $\mathrm{PaO}_{2} / \mathrm{FiO}_{2}$ ratio was sustained even after the patients returned to the supine position[42,45,46]; one study $(n=46)$ demonstrated sustained improvement in only $50 \%$ of patients[14], and in another report $(n=26)$, improvement of $\mathrm{PaO}_{2} / \mathrm{FiO}_{2}$ was lost after returning to supine position[43].

Reduction of respiratory rate with APP was demonstrated in five studies $(n=90)[10,28,36,40,41]$, but not in two other studies $(n=34)[33,45]$. Finally, significantly reduced $\mathrm{PaCO}_{2}$ was demonstrated only in a single small study $(n=9)$ [35], while no changes in $\mathrm{PaCO}_{2}$ were observed in a larger report $(\mathrm{n}=46)[14]$.

\section{Probability of intubation with awake prone positioning}

Data on intubation rate were available for 870 patients treated with APP ( 23 published studies, $n=717 ; 2$ unpublished studies, $n=153$ ), and for 852 patients treated with HFNC, or CPAP, or NIV, without APP (16 published studies, $n=645 ; 2$ unpublished studies, $n=207)$. In the APP group, $27 \%(95 \% \mathrm{Cl}, 19$ to $37 \%)$ required intubation and mechanical ventilation, as 
compared to $30 \%(95 \% \mathrm{Cl}, 20$ to $42 \%)$ in the control group (Figure 2). This difference was not statistically significant $(p=0.71)$.

Subgroup analyses, with stratification according to the duration of APP ( $<4 \mathrm{~h}$ daily vs $\geq 4 \mathrm{~h}$ daily), the device (HFNC vs CPAP vs NIV), and the severity of the ARDS $\left(\mathrm{PaO}_{2} / \mathrm{FiO}_{2}<150 \mathrm{mmHg}\right.$ vs $\left.\mathrm{PaO}_{2} / \mathrm{FiO}_{2} \geq 150 \mathrm{mmHg}\right)$ did not demonstrate any significant difference in intubation rate between patients who were treated with APP and those who were not (Figure 3 ).

There was a very high level of heterogeneity across all studies. Sensitivity analyses with the inclusion of the subgroup of patients with some exposure to APP, and with exclusion of unpublished data demonstrated a similar lack of benefit (Figures E3 through E11), as did the analyses according to country GDP (Figures E23 and E24).

\section{Probability of death with awake prone positioning}

Mortality data were available for 767 patients treated with APP (18 published studies, $n=614 ; 2$ unpublished studies, $\mathrm{n}=153$ ) and for 761 patients treated with HFNC, or CPAP, or NIV, without APP (12 published studies, $\mathrm{n}=554 ; 2$ unpublished studies, $n=207)$. The mortality rate was $11 \%(95 \% \mathrm{Cl}, 6$ to $20 \%)$ in patients treated with APP, as compared to $22 \%(95 \% \mathrm{Cl}, 13$ to $36 \%$ ) in patients treated with usual care (Figure 4). This difference was not statistically significant $(p=0.10)$.

Outcomes were highly heterogeneous between studies, and subgroup analyses did not demonstrate any significant differences in mortality across predetermined subgroups (Figure 5), and did not identify a subgroup in which APP was associated with statistically significant reduction of mortality. Sensitivity analyses with the inclusion of patients with partial exposure to APP, with exclusion of unpublished data, and with exclusion of patients treated with conventional oxygen therapy demonstrated a similar lack of mortality benefit (Figures E12 through E22). No statistically significant signal was seen in analyses restricted to high- or low-GDP countries (Figures E25 and E26)

\section{Tolerability and comfort of awake prone positioning}

Fifteen studies reported patients' tolerability to APP, varying from $47 \%$ to $100 \%[9,10,12,14,28-30,32,33,35,36,40,43,45,46]$. Eight papers reported on patient's discomfort while in prone position[9,12,14,32,36,40,43,45], including back pain, dyspnea, and general discomfort. The daily duration of APP was reported in 17 papers $(n=366)[8-$

$10,12,14,28,29,31,34,36,38,40,41,45-47]$. In 9 papers ( $n=201)$, patients tolerated APP for less than 4 hours daily[8$10,14,28,31,38,40,41]$. A single paper reported on a cohort of 55 patients who were able to achieve APP for more than 16 hours daily[47].

\section{Discussion}

Our systematic review and meta-analysis demonstrated that APP improved oxygenation but did not change the frequency of intubation or mortality in patients with AHRF secondary to COVID-19. The main strength of our study was the large sample size, with a total of 921 patients treated with APP. However, given the observational nature of included studies, high heterogeneity, and broad confidence intervals, our results should be interpreted as demonstrating absence of evidence, rather than high quality evidence of an absence of benefit.

We found that APP improved oxygenation parameters, and this improvement was sustained even after the patients returned to the supine position in three studies[42,45,46]. APP was also associated with reduced respiratory rate, and good tolerability was reported with the use of various modalities of respiratory support, including conventional oxygen therapy, HFNC, and CPAP or and NIV that was delivered through either a helmet or full face mask. Improvement in oxygenation with APP can be explained by the correction of ventilation/perfusion mismatch[11], better lung recruitment, and reduction of 
alveolar strain[17]. However, improvements in oxygenation do not guarantee better clinical outcomes. For instance, improvements of $\mathrm{PaO}_{2} / \mathrm{FiO}_{2}$ ratio do not correlate with mortality in intubated patients subjected to prone positioning[69]. More physiological and clinical studies are needed to delineate the relationship between improvement of oxygenation parameters and clinical outcomes in patients with COVID-19.

Contrary to previous reports[19,34], we did not find that APP reduced intubation rates. Several reasons can be advanced to explain this lack of efficacy. First, intubation criteria were not uniformly defined across studies, and involved the treating physician's subjective judgment. During the pandemic, the recommended respiratory support strategies evolved from early aggressive intubation to strategies of respiratory support designed to prevent intubation[8,55,70-72]. Second, the timing of APP initiation, either as an "adjunctive" (early) or "salvage" (late) therapy may influence intubation rate. The use of APP at an early stage $\left(\mathrm{PaO}_{2} / \mathrm{FiO}_{2}\right.$ ratio $\left.>150 \mathrm{mmHg}\right)$ may be better tolerated, result in better oxygenation, and protect patients from self-induced lung injury (SILI), and thus prevent further disease progression[73,74]. However, in our meta-analysis, we did not detect a signal of benefit of APP in the subgroup of patients with $\mathrm{PaO}_{2} / \mathrm{FiO}_{2}$ ratio $>150 \mathrm{mmHg}$. Third, the duration of APP might have a dose-response relationship, and it is possible that a reduction in the rate of intubations could be seen only in patients who were subjected to longer periods of APP. Our subgroup analyses did not demonstrate significantly lower intubation rates for patients who remained in PP for longer periods of time, but it could be argued that our analysis was underpowered, as only two studies $(n=65)$ reported daily APP periods $>16 h[12,47]$. Fourth, intubation might be inevitable as the disease progresses, despite initial and sustained improvement in oxygenation. It has been argued that intubation rates are lower in patients who experience sustained improvement in oxygenation after APP, the so-called "responders"[46]. However, this finding has not been replicated in other retrospective studies[14], and could be the result of simple reverse causality, with patients "responding" to APP because of their already favorable clinical course. Finally, an unknown proportion of patients with do-not-intubate orders were included in both groups, which could have diluted any possible benefit of APP.

We did not demonstrate a signal of reduced mortality with APP. Given the complex relationship between disease severity, individual co-morbidities, socio-economic status, and variable access to quality care during a pandemic, this finding should be interpreted as exploratory. Due to the retrospective nature of included studies, selection biases are very likely. The type of respiratory support (conventional oxygen therapy, HFNC, CPAP/NIV delivered through a conventional mask vs a helmet) was not balanced between patients treated with APP and those who were not. Analyses with stratification by the type of respiratory support device did not demonstrate significant subgroup differences in mortality. These subgroup analyses were severely limited by the fact that we had access only to overall group statistics, not individual patient data, and a proportion of patients were treated with various devices through the course of their disease.

The use of APP has been widely promoted as the new standard of care, and even included as such clinical care guidelines[76], on the basis of physiological plausibility and low quality evidence from case reports. It has been suggested that studies of this intervention are not warranted and that investigators lack equipoise. Our meta-analysis of available low quality data does not support this unbridled enthusiasm, and we strongly caution against uncritical inclusion of unproven therapies in routine patient care. The critical care literature is replete with examples of interventions that improved surrogate outcomes, yet ultimately failed to improve clinical outcomes in randomized controlled trials[77-79]. Implementing an ineffective therapy is not benign, as, at best, this diverts finite clinical resources and attention. At worst, it could result in unnecessarily delayed intubations in difficult conditions, self-induced lung injury, and potentially higher mortality[80]. Unfortunately, there are numerous precedents of physiologically sound therapies that unexpectedly resulted in higher mortality, such as aggressive fluid resuscitation[81] or liberal red-cell transfusions[82].

\section{Limitations}


Our study has several significant limitations. First, data were available only from a group of relatively heterogeneous observational studies. Significant levels of inclusion bias are also likely to be present. Without individual patient data, we could not account for the many uncontrolled differences between patients treated with APP, and those who received usual care. Some patients were subjected to APP in extremis after failing usual care, and could have been sicker than patients included in cohorts without APP. Conversely, in other reports, only patients who could self-prone were treated with APP, and these were likely less sick than those in the control group. Second, a variety of respiratory support devices, including helmet CPAPs, were used in both groups. It is not known whether the choice of the device has an impact on outcomes in patients with severe COVID-19. Third, outcomes were highly heterogeneous, which likely reflects populations with various disease severities, various co-morbid conditions, as well as geographical variations of care for patients with ARDS[75]. Fourth, we included unpublished, non-peer-reviewed data. However, our findings remained robust with the exclusion of unpublished data. Fifth, the mortality rate in our studies is lower than reported in other large cohorts[2,4,5], which suggests selection and publications bias, which would be expected to be in favor of APP. Sixth, we were not able to control for the use of evidence-based treatments such as corticosteroids. However, all included reports finished enrolment before the benefit of corticosteroid was demonstrated [83] and when their use was indeed actively discouraged. Seventh, only a minority of patients were able to tolerate longer periods of APP, and it can be argued that the duration of APP was not sufficient to generate a clinically meaningful change in outcomes. However, a physiologically effective, but clinically intolerable intervention would remain ineffective overall. Lastly, data for other important outcomes, such the number of ventilator-free days or the length of ICU stay, were not available for analysis.

\section{Conclusions}

In summary, available evidence from observational studies suggests that awake prone positioning improves oxygenation, but these improvements do not appear to translate into reduced rates of intubation. We did not find any obvious signals of harm, and we did not see any worrisome signal in mortality.

The high selectivity of patients, the inconsistency in the application of prone positioning in published reports and the heterogeneity of outcomes emphasizes the need for randomized controlled trials, as a clinically significant benefit cannot be excluded based on available low-quality data.

\section{List Of Abbreviations}

Awake prone positioning (APP); coronavirus disease 2019 (COVID-19); acute hypoxemic respiratory failure (AHRF); acute respiratory distress syndrome (ARDS); intensive care unit (ICU); mechanical ventilation (MV); extracorporeal membrane oxygenation (ECMO); high-flow nasal cannula (HFNC); non-invasive ventilation (NIV); Continuous positive airway pressure (CPAP); arterial oxygen partial pressure $\left(\mathrm{PaO}_{2}\right)$; fraction of inspired oxygen $\left(\mathrm{FiO}_{2}\right)$; confidence interval $(\mathrm{Cl})$; Peripheral oxygen saturation $\left(\mathrm{SpO}_{2}\right)$; self-induced lung injury (SILI).

\section{Declarations}

Ethics approval and consent to participate

All published and unpublished reports included in the meta-analysis have undergone appropriate ethical approval.

Consent for publication

Not applicable

Availability of data and materials

Not applicable 
JL has received research support from Fisher \& Paykel Healthcare Ltd, Aerogen Ltd, and Rice Foundation, and lecture honorarium from AARC and Fisher \& Paykel Healthcare Ltd outside the submitted work.

IP received a research grant and speaker fees from Fisher \& Paykel Healthcare.

SE and YP received research support from Fisher \& Paykel Healthcare.

OR provides consultancy to Hamilton Medical. All fees were received by his Institution of Research. He also received speaker fees by Air Liquide.

JGL has received research grant and consulting fees from Baxter Healthcare.

SE received unrestricted research grants, travel fee reimbursements and speaker fees from Fisher \& Paykel Healthcare, consulting fees from La Diffusion Technique Française, consulting fees and unrestricted research grants from Aerogen Ltd., and an unrestricted research grant from Hamilton medical.

JAJ and MWT received speaker fees from Fisher \& Paykel Healthcare

DR is the president of DRDR Mobile Health, a company that creates mobile applications for healthcare, including functional capacity assessment applications. He has engaged in consulting for mobile applications as well. He has not taken any salary or money from the company.

DLV reports consulting for Ohio Medical, speaking for Theravance Biopharma, and research funding from Teleflex Medical, Inc. and Rice Foundation.

Other authors have no conflict of interest to declare.

Funding

This work was supported by OpenAl, 3180 18th St, San Francisco, CA 94110, United States and by Rice Foundation, 8600 Gross Point Rd, Skokie, IL 60077. The funding bodies had no role in the design of the study, in collection, analysis, and interpretation of data, nor in writing of the manuscript.

Authors' contributions

$\mathrm{BL}, \mathrm{HH}, \mathrm{IP}, \mathrm{JL}$, and YP extracted and summarized data from individual papers.

ET performed the statistical analyses

IP, HH, JL drafted the manuscript

All authors provided critical feedback and reviewed the manuscript.

Acknowledgements

Not applicable

\section{References}

1. Ziehr DR, Alladina J, Petri CR, et al. Respiratory pathophysiology of mechanically ventilated patients with COVID-19: A cohort study. American Journal of Respiratory and Critical Care Medicine. 2020;201(12):1560-1564. doi:10.1164/rccm.202004-1163LE

2. Intensive care national audit and research centre. ICNARC report on COVID-19 in critical care 31 july 2020. Available at https://wwwicnarcorg/DataServices/Attachments/Download/42ceb4d2-3dd3-ea11-9128-00505601089b.

3. Grasselli G, Greco M, Zanella A, et al. Risk Factors Associated With Mortality Among Patients With COVID-19 in Intensive Care Units in Lombardy, Italy. JAMA Internal Medicine. 2020;180(10):1345-1355.

Page $10 / 20$ 
doi:10.1001/jamainternmed.2020.3539

4. Auld SC, Caridi-Scheible M, Blum JM, et al. ICU and ventilator mortality among critically ill adults with coronavirus disease 2019. Critical Care Medicine. 2020;48(9).

https://journals.Iww.com/ccmjournal/Fulltext/2020/09000/ICU_and_Ventilator_Mortality_Among_Critically_III.35.aspx

5. Karagiannidis C, Mostert C, Hentschker C, et al. Case characteristics, resource use, and outcomes of 10021 patients with COVID-19 admitted to 920 German hospitals: An observational study. The Lancet Respiratory Medicine. 2020;8(9):853-862.

6. Fagiuoli S, Lorini FL, Remuzzi G. Adaptations and lessons in the province of Bergamo. New England Journal of Medicine. 2020;382(21):e71.

7. Sun Q, Qiu H, Huang M, Yang Y. Lower mortality of COVID-19 by early recognition and intervention: Experience from Jiangsu province. Annals of Intensive Care. 2020;10(1):33. doi:10.1186/s13613-020-00650-2

8. Caputo ND, Strayer RJ, Levitan R. Early self-proning in awake, non-intubated patients in the emergency department: A single ED's experience during the COVID-19 pandemic. Academic Emergency Medicine. 2020;27(5):375-378.

9. Elharrar X, Trigui Y, Dols A-M, et al. Use of Prone Positioning in Nonintubated Patients With COVID-19 and Hypoxemic Acute Respiratory Failure. JAMA. 2020;323(22):2336-2338. doi:10.1001/jama.2020.8255

10. Sartini C, Tresoldi M, Scarpellini P, et al. Respiratory Parameters in Patients With COVID-19 After Using Noninvasive Ventilation in the Prone Position Outside the Intensive Care Unit. JAMA. 2020;323(22):2338-2340. doi:10.1001/jama.2020.7861

11. Zarantonello F, Andreatta G, Sella N, Navalesi P. Prone position and lung ventilation and perfusion matching in acute respiratory failure due to COVID-19. American journal of respiratory and critical care medicine. 2020;202(2):278-279. doi:10.1164/rccm.202003-0775IM

12. Xu Q, Wang T, Qin X, Jie Y, Zha L, Lu W. Early awake prone position combined with high-flow nasal oxygen therapy in severe COVID-19: A case series. Critical Care. 2020;24(1):250. doi:10.1186/s13054-020-02991-7

13. Slessarev M, Cheng J, Ondrejicka M, Arntfield R, Group CCWR. Patient self-proning with high-flow nasal cannula improves oxygenation in COVID-19 pneumonia. Canadian journal of anaesthesia = Journal canadien d'anesthesie. 2020;67(9):1288-1290. doi:10.1007/s12630-020-01661-0

14. Coppo A, Bellani G, Winterton D, et al. Feasibility and physiological effects of prone positioning in non-intubated patients with acute respiratory failure due to COVID-19 (PRON-COVID): A prospective cohort study. The Lancet Respiratory Medicine. 2020;8(8):765-774.

15. Guérin C, Reignier J, Richard J-C, et al. Prone positioning in severe acute respiratory distress syndrome. New England Journal of Medicine. 2013;368(23):2159-2168.

16. Sud S, Friedrich JO, Adhikari NKJ, et al. Effect of prone positioning during mechanical ventilation on mortality among patients with acute respiratory distress syndrome: A systematic review and meta-analysis. CMAJ. 2014;186(10):E381E390. doi:10.1503/cmaj.140081

17. Kallet RH. A comprehensive review of prone position in ARDS. Respiratory care. 2015;60(11):1660-1687.

18. Scaravilli V, Grasselli G, Castagna L, et al. Prone positioning improves oxygenation in spontaneously breathing nonintubated patients with hypoxemic acute respiratory failure: A retrospective study. Journal of Critical Care. 2015;30(6):1390-1394

19. Ding L, Wang L, Ma W, He H. Efficacy and safety of early prone positioning combined with HFNC or NIV in moderate to severe ARDS: A multi-center prospective cohort study. Critical Care. 2020;24(1):28. doi:10.1186/s13054-020-2738-5

20. Moher D, Liberati A, Tetzlaff J, Altman DG, Group P, others. Preferred reporting items for systematic reviews and metaanalyses: The PRISMA statement. PLoS med. 2009;6(7):e1000097. 
21. Lemasson S, Ayzac L, Girard R, Gaillard S, Pavaday K, Guérin C. Does gas exchange response to prone position predict mortality in hypoxemic acute respiratory failure? Intensive care medicine. 2006;32(12):1987-1993.

22. Rice TW, Wheeler AP, Bernard GR, et al. Comparison of the SpO2/FiO2 ratio and the $\mathrm{PaO} 2 / \mathrm{FiO} 2$ ratio in patients with acute lung injury or ARDS. Chest. 2007;132(2):410-417.

23. Chen W, Janz DR, Shaver CM, Bernard GR, Bastarache JA, Ware LB. Clinical characteristics and outcomes are similar in ARDS diagnosed by oxygen saturation/FiO2 ratio compared with PaO2/FiO2 ratio. Chest. 2015;148(6):1477-1483.

24. Bellani G, Laffey JG, Pham T, et al. Noninvasive ventilation of patients with acute respiratory distress syndrome. insights from the LUNG SAFE study. American Journal of Respiratory and Critical Care Medicine. 2017;195(1):67-77. doi:10.1164/rccm.201606-13060C

25. Hunter JP, Saratzis A, Sutton AJ, Boucher RH, Sayers RD, Bown MJ. In meta-analyses of proportion studies, funnel plots were found to be an inaccurate method of assessing publication bias. Journal of clinical epidemiology. 2014;67(8):897-903.

26. R Core Team. R: A Language and Environment for Statistical Computing. R Foundation for Statistical Computing; 2017. https://www.R-project.org/

27. Balduzzi S, Rücker G, Schwarzer G. How to perform a meta-analysis with R: A practical tutorial. Evidence-Based Mental Health. 2019;(22):153-160.

28. Damarla M, Zaeh S, Niedermeyer S, et al. Prone positioning of nonintubated patients with COVID-19. American journal of respiratory and critical care medicine. 2020;202(4):604-606.

29. Despres C, Brunin Y, Berthier F, Pili-Floury S, Besch G. Prone positioning combined with high-flow nasal or conventional oxygen therapy in severe COVID-19 patients. Critical Care. 2020;24(1):256. doi:10.1186/s13054-020-03001-6

30. Golestani-Eraghi M, Mahmoodpoor A. Early application of prone position for management of COVID-19 patients. Journal of clinical anesthesia. 2020;66:109917-109917. doi:10.1016/j.jclinane.2020.109917

31. Huang C-F, Tay CK, Zhuang Y-F, Liu J, Sewa DW. Rationale and significance of patient selection in awake prone positioning for COVID-19 pneumonia. European Respiratory Journal. 2020;56(3). doi:10.1183/13993003.02173-2020

32. Ng Z, Tay WC, Ho CHB. Awake prone positioning for non-intubated oxygen dependent COVID-19 pneumonia patients. European Respiratory Journal. Published online 2020. doi:10.1183/13993003.01198-2020

33. Moghadam VD, Shafiee H, Ghorbani M, Heidarifar R. Prone positioning in management of COVID-19 hospitalized patients. Brazilian journal of anesthesiology (Elsevier). 2020;70(2):188-190. doi:10.1016/j.bjane.2020.05.001

34. Thompson AE, Ranard BL, Wei Y, Jelic S. Prone positioning in awake, nonintubated patients with COVID-19 hypoxemic respiratory failure. JAMA internal medicine. Published online Jun 17, 2020:e203030.

doi:10.1001/jamainternmed.2020.3030

35. Tu G-W, Liao Y-X, Li Q-Y, et al. Prone positioning in high-flow nasal cannula for COVID-19 patients with severe hypoxemia: A pilot study. Annals of Translational Medicine. 2020;8(9). http://atm.amegroups.com/article/view/41073

36. Dong W, Gong Y, Feng J, et al. Early awake prone and lateral position in non-intubated severe and critical patients with COVID-19 in Wuhan: A respective cohort study. medRxiv. Published online 2020. doi:10.1101/2020.05.09.20091454

37. Bastoni D, Poggiali E, Vercelli A, et al. Prone positioning in patients treated with non-invasive ventilation for COVID-19 pneumonia in an Italian emergency department. Emergency medicine journal : EMJ. 2020;37(9):565-566.

doi:10.1136/emermed-2020-209744

38. Ripoll-Gallardo A, Grillenzoni L, Bollon J, Della Corte F, Barone-Adesi F. Prone positioning in non-intubated patients with COVID-19 outside of the intensive care unit: More evidence needed. Disaster medicine and public health preparedness. Published online Jul 27, 2020:1-3. doi:10.1017/dmp.2020.267

39. Villarreal-Fernandez E, Patel R, Golamari R, Khalid M, DeWaters A, Haouzi P. A plea for avoiding systematic intubation in severely hypoxemic patients with COVID-19-associated respiratory failure. Critical care. 2020;24(1):337-337. 
doi:10.1186/s13054-020-03063-6

40. Solverson K, Weatherald J, Parhar KKS. Tolerability and safety of awake prone positioning COVID-19 patients with severe hypoxemic respiratory failure. Canadian journal of anaesthesia = Journal canadien d'anesthesie. Published online Aug 14, 2020:1-7. doi:10.1007/s12630-020-01787-1

41. Zang X, Wang Q, Zhou H, et al. Efficacy of early prone position for COVID-19 patients with severe hypoxia: A singlecenter prospective cohort study. Intensive Care Medicine. 2020;46(10):1927-1929. doi:10.1007/s00134-020-06182-4

42. Taboada M, Rodríguez N, Riveiro V, Baluja A, Atanassoff PG. Prone positioning in awake non-ICU patients with ARDS caused by COVID-19. Anaesthesia, critical care \& pain medicine. 2020;39(5):581-583. doi:10.1016/j.accpm.2020.08.002

43. Retucci M, Aliberti S, Ceruti C, et al. Prone and lateral positioning in spontaneously breathing patients with COVID-19 pneumonia undergoing noninvasive helmet CPAP treatment. Chest. Published online Jul 15, 2020:S00123692(20)31888-2. doi:10.1016/j.chest.2020.07.006

44. Calligaro GL, Lalla U, Audley G, et al. The utility of high-flow nasal oxygen for severe COVID-19 pneumonia in a resource-constrained setting: A multi-centre prospective observational study. EClinicalMedicine. doi:10.1016/j.eclinm.2020.100570

45. Winearls S, Swingwood EL, Hardaker CL, et al. Early conscious prone positioning in patients with COVID-19 receiving continuous positive airway pressure: A retrospective analysis. BMJ Open Respiratory Research. 2020;7(1). doi:10.1136/bmjresp-2020-000711

46. Burton-Papp HC, Jackson Al, Beecham R, et al. Conscious prone positioning during non-invasive ventilation in COVID19 patients: Experience from a single centre. F1000Research. 2020;9(859):859.

47. Ferrando C, Mellado-Artigas R, Gea A, et al. Awake prone positioning does not reduce the risk of intubation in COVID19 treated with high-flow nasal oxygen therapy: A multicenter, adjusted cohort study. Critical Care. 2020;24(1):597. doi:10.1186/s13054-020-03314-6

48. Geng S, Mei Q, Zhu C, et al. High flow nasal cannula is a good treatment option for COVID-19. Heart \& lung : the journal of critical care. 2020;49(5):444-445. doi:10.1016/j.hrtlng.2020.03.018

49. Wang K, Zhao W, Li J, Shu W, Duan J. The experience of high-flow nasal cannula in hospitalized patients with 2019 novel coronavirus-infected pneumonia in two hospitals of chongqing, china. Annals of intensive care. 2020;10(1):3737. doi:10.1186/s13613-020-00653-z

50. Patel M, Gangemi A, Marron R, et al. Retrospective analysis of high flow nasal therapy in COVID-19-related moderateto-severe hypoxaemic respiratory failure. BMJ Open Respiratory Research. 2020;7(1). doi:10.1136/bmjresp-2020000650

51. Vianello A, Arcaro G, Molena B, et al. High-flow nasal cannula oxygen therapy to treat patients with hypoxemic acute respiratory failure consequent to SARS-CoV-2 infection. Thorax. 2020;75(11):998-1000. doi:10.1136/thoraxjnl-2020214993

52. Zucman N, Mullaert J, Roux D, et al. Prediction of outcome of nasal high flow use during COVID-19-related acute hypoxemic respiratory failure. Intensive Care Medicine. 2020;46(10):1924-1926. doi:10.1007/s00134-020-06177-1

53. Blez D, Soulier A, Bonnet F, Gayat E, Garnier M. Monitoring of high-flow nasal cannula for SARS-CoV-2 severe pneumonia: Less is more, better look at respiratory rate. Intensive care medicine. Published online Jul 31, 2020:1-2. doi:10.1007/s00134-020-06199-9

54. Xia J, Zhang Y, Ni L, et al. High-flow nasal oxygen in coronavirus disease 2019 patients with acute hypoxemic respiratory failure: A multicenter, retrospective cohort study. Critical care medicine. 2020;48(11):e1079-e1086. doi:10.1097/CCM.0000000000004558

55. Hernandez-Romieu AC, Adelman MW, Hockstein MA, et al. Timing of intubation and mortality among critically ill coronavirus disease 2019 patients: A single-center cohort study. Critical care medicine. 2020;48(11):e1045-e1053. 
doi:10.1097/CCM.0000000000004600

56. He G, Han Y, Fang Q, et al. Clinical experience of high-flow nasal cannula oxygen therapy in severe COVID-19 patients. Journal of Zhejiang University (Medical Science). 2020;49(2):232-239.

57. Pagano A, Porta G, Bosso G, et al. Non-invasive CPAP in mild and moderate ARDS secondary to SARS-CoV-2. Respiratory physiology \& neurobiology. 2020;280:103489-103489. doi:10.1016/j.resp.2020.103489

58. Burns GP, Lane ND, Tedd HM, et al. Improved survival following ward-based non-invasive pressure support for severe hypoxia in a cohort of frail patients with COVID-19: Retrospective analysis from a UK teaching hospital. BMJ open respiratory research. 2020;7(1):e000621. doi:10.1136/bmjresp-2020-000621

59. Oranger M, Gonzalez-Bermejo J, Dacosta-Noble P, et al. Continuous positive airway pressure to avoid intubation in SARS-CoV-2 pneumonia: A two-period retrospective case-control study. The European respiratory journal. 2020;56(2):2001692. doi:10.1183/13993003.01692-2020

60. Knights H, Mayor N, Millar K, et al. Characteristics and outcomes of patients with COVID-19 at a district general hospital in Surrey, UK. Clinical medicine (London, England). 2020;20(5):e148-e153. doi:10.7861/clinmed.2020-0303

61. Duca A, Memaj I, Zanardi F, et al. Severity of respiratory failure and outcome of patients needing a ventilatory support in the emergency department during Italian novel coronavirus SARS-CoV-2 outbreak: Preliminary data on the role of helmet CPAP and non-invasive positive pressure ventilation. EClinicalMedicine. 2020;24:100419-100419. doi:10.1016/j.eclinm.2020.100419

62. Sivaloganathan AA, Nasim-Mohi M, Brown MM, et al. Noninvasive ventilation for COVID-19-associated acute hypoxaemic respiratory failure: Experience from a single centre. British Journal of Anaesthesia. 202010AD;125(4):e368-e371. doi:10.1016/j.bja.2020.07.008

63. Hallifax RJ, Porter BM, Elder PJ, et al. Successful awake proning is associated with improved clinical outcomes in patients with COVID-19: Single-centre high-dependency unit experience., ed. BMJ Open Respiratory Research. 2020;7(1). doi:10.1136/bmjresp-2020-000678

64. Franco C, Facciolongo N, Tonelli R, et al. Feasibility and clinical impact of out-of-ICU non-invasive respiratory support in patients with COVID-19 related pneumonia. The European respiratory journal. Published online Aug 03, 2020:2002130. doi:10.1183/13993003.02130-2020

65. Demoule A, Vieillard Baron A, Darmon M, et al. High-flow nasal cannula in critically ill patients with severe COVID-19. American journal of respiratory and critical care medicine. 2020;202(7):1039-1042. doi:10.1164/rccm.202005-2007LE

66. Aliberti S, Radovanovic D, Billi F, et al. Helmet CPAP treatment in patients with COVID-19 pneumonia: A multicentre cohort study. The European respiratory journal. 2020;56(4):2001935. doi:10.1183/13993003.01935-2020

67. Nightingale R, Nwosu N, Kutubudin F, et al. Is continuous positive airway pressure (CPAP) a new standard of care for type 1 respiratory failure in COVID-19 patients? A retrospective observational study of a dedicated COVID-19 CPAP service. BMJ Open Respiratory Research. 2020;7(1). doi:10.1136/bmjresp-2020-000639

68. Panadero C, Abad-Fernández A, Rio-Ramirez MT, et al. High-flow nasal cannula for acute respiratory distress syndrome (ARDS) due to COVID-19. Multidisciplinary respiratory medicine. 2020;15(1):693-693. doi:10.4081/mrm.2020.693

69. Albert RK, Keniston A, Baboi L, Ayzac L, Guérin C. Prone position-induced improvement in gas exchange does not predict improved survival in the acute respiratory distress syndrome. American journal of respiratory and critical care medicine. 2014;189(4):494-496.

70. Colla J, Rodos A, Seyller H, Weingart S. Fighting COVID-19 hypoxia with one hand tied behind our back: Blanket prohibition of high-flow oxygen and noninvasive positive end-expiratory pressure in uS hospitals. Annals of Emergency Medicine. 2020-6AD;75(6):791-792. doi:10.1016/j.annemergmed.2020.04.015

71. Li J, Fink JB, Ehrmann S. High-flow nasal cannula for COVID-19 patients: Low risk of bio-aerosol dispersion. European Respiratory Journal. 2020;55(5). doi:10.1183/13993003.00892-2020

Page $14 / 20$ 
72. Whittle JS, Pavlov I, Sacchetti AD, Atwood C, Rosenberg MS. Respiratory support for adult patients with COVID-19. Journal of the American College of Emergency Physicians Open. 2020;1(2):95-101.

73. Brochard L, Slutsky A, Pesenti A. Mechanical ventilation to minimize progression of lung injury in acute respiratory failure. American journal of respiratory and critical care medicine. 2017;195(4):438-442.

74. Cruces P, Retamal J, Hurtado DE, et al. A physiological approach to understand the role of respiratory effort in the progression of lung injury in SARS-CoV-2 infection. Critical Care. 2020;24(1):1-10.

75. Laffey JG, Madotto F, Bellani G, et al. Geo-economic variations in epidemiology, patterns of care, and outcomes in patients with acute respiratory distress syndrome: Insights from the LUNG SAFE prospective cohort study. The Lancet Respiratory Medicine. 2017;5(8):627-638.

76. Bamford P, Bentley A, Dean J, Whitmore D, Wilson-Baig N. ICS guidance for prone positioning of the conscious COVID patient 2020. Available at https://wwwicnarcorg/DataServices/Attachments/Download/42ceb4d2-3dd3-ea11-9128$00505601089 \mathrm{~b}$.

77. Lundin S, Mang H, Smithies M, Stenqvist O, Frostell C, others. Inhalation of nitric oxide in acute lung injury: Results of a European multicentre study. Intensive care medicine. 1999;25(9):911-919.

78. Liu KD, Levitt J, Zhuo H, et al. Randomized clinical trial of activated protein $\mathrm{C}$ for the treatment of acute lung injury. American journal of respiratory and critical care medicine. 2008;178(6):618-623.

79. National Heart Lung, Network BIACT. Rosuvastatin for sepsis-associated acute respiratory distress syndrome. New England Journal of Medicine. 2014;370(23):2191-2200.

80. Walkey AJ, Wiener RS. Use of noninvasive ventilation in patients with acute respiratory failure, 2000-2009: A population-based study. Annals of the American Thoracic Society. 2013;10(1):10-17.

81. Bickell WH, Wall Jr MJ, Pepe PE, et al. Immediate versus delayed fluid resuscitation for hypotensive patients with penetrating torso injuries. New England Journal of Medicine. 1994;331(17):1105-1109.

82. Hébert PC, Wells G, Blajchman MA, et al. A multicenter, randomized, controlled clinical trial of transfusion requirements in critical care. New England Journal of Medicine. 1999;340(6):409-417.

83. The RECOVERY Collaborative Group. Dexamethasone in hospitalized patients with Covid-19 - preliminary report. N Engl J Med. DOI: 10.1056/NEJMoa2021436.

\section{Table}

\section{Due to technical limitations, table $1 \mathrm{xlsx}$ is only available as a download in the Supplemental Files section.}

Table 1. Summary of included studies of patients severe COVID treating with, and without the use of awake prone positioning.

Abbreviations, in order of appearance: $\mathrm{P} / \mathrm{F}, \mathrm{PaO}_{2} / \mathrm{FiO}_{2}$, i.e., the ratio of arterial oxygen partial pressure $(\mathrm{PaO}$ in $\mathrm{mmHg}$ to fractional inspired oxygen $\left(\mathrm{FiO}_{2}\right) ; \mathrm{PaCO}_{2}$, arterial carbon dioxide partial pressure; $\mathrm{SpO}_{2}$, peripheral oxygen saturation; $\mathrm{ED}$, emergency department; IMCU, intermediate care unit; ICU, intensive care unit; CPAP, Continuous positive airway pressure; COT, conventional oxygen therapy; NIV, non-invasive ventilation; APP, awake prone positioning.

The data on the prevalence and the duration of APP included unpublished data obtained by personal communication with principal investigators of included studies. Continuous variables are reported as means or medians. Missing variables are marked with a dash. Whenever possible, mortality is reported only for the subset of patients discharged from the ICU at the moment of each study publication. Outcomes are reported stratified by the type of respiratory support device.

a The papers by Retucci et al., and Burton-Papp et al., report on the subset populations of patients from larger cohorts 
described by Aliberti et al., and Sivaloganathan et al., respectively. In order to avoid counting single patients twice, we report data from the two larger cohorts with the exclusion of outcomes of patients managed with APP.

${ }^{b}$ Burns et al. report on a cohort comprised exclusively by patients with do-not-intubate orders, which was not included in the meta-analysis of the intubation rate.

\section{Figures}

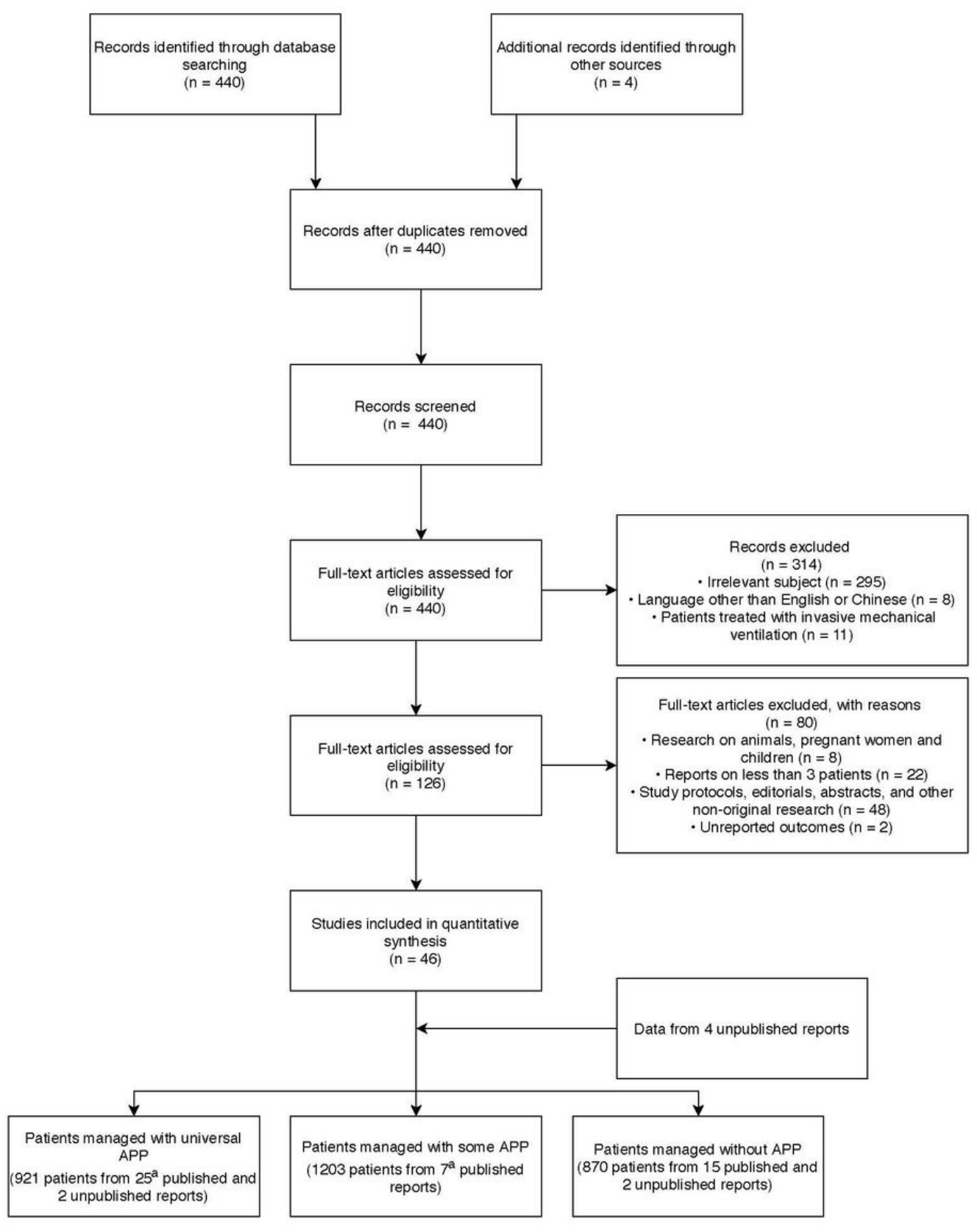

\section{Figure 1}

Flow diagram showing identification of eligible studies included in the meta-analysis. aOne paper reported on both subgroups, and is thus counted twice. 
No prone positioning

Blez et al, 2020

Burns et al, 2020

Duca et al, 2020

Geng et al, 2020

$\mathrm{He}$ et al, 2020

Hernandez-Romieu et al, 2020

Knights et al, 2020

Oranger et al, 2020

Patel et al, 2020

Sivaloganathan et al, 2020

Vianello et al, 2020

Wang et al, 2020

Xia et al, 2020

Zucman et al, 2020

Unpublished data from UChicago Medicine

Random effects model

Heterogeneity: $I^{2}=90 \%, \tau^{2}=0.9432, p<0.01$

Prone positioning

Bastoni et al, 2020

Burton-Papp et al, 2020

Calligaro et al, 2020

Caputo et al, 2020

Coppo et al, 2020

Damarla et al, 2020

Despres et al, 2020

Dong et al, 2020 (Preprint)

Elharrar et al, 2020

Ferrando et al, 2020

Golestani-Eraghi et al, 2020

Huang et al, 2020

Moghadam et al, 2020

$\mathrm{Ng}$ et al, 2020

Retucci et al., 2020

Ripoll-Gallardo et al, 2020

Sartini et al, 2020

Solverson et al, 2020

Thompson et al, 2020

Tu et al, 2020

Villarreal-Fernandez et al, 2020

Winearls et al, 2020

Xu et al, 2020

Unpublished data from the Irish awake proning group Unpublished data from Unity Point Health

Random effects model

Heterogeneity: $I^{2}=85 \%, \tau^{2}=0.9138, p<0.01$

Heterogeneity: $I^{2}=88 \%, \tau^{2}=0.9311, p<0.01$

Test for subgroup differences: $\chi_{1}^{2}=0.14, \mathrm{df}=1(p=0.71)$
Unpublished data from Duan et al (China)
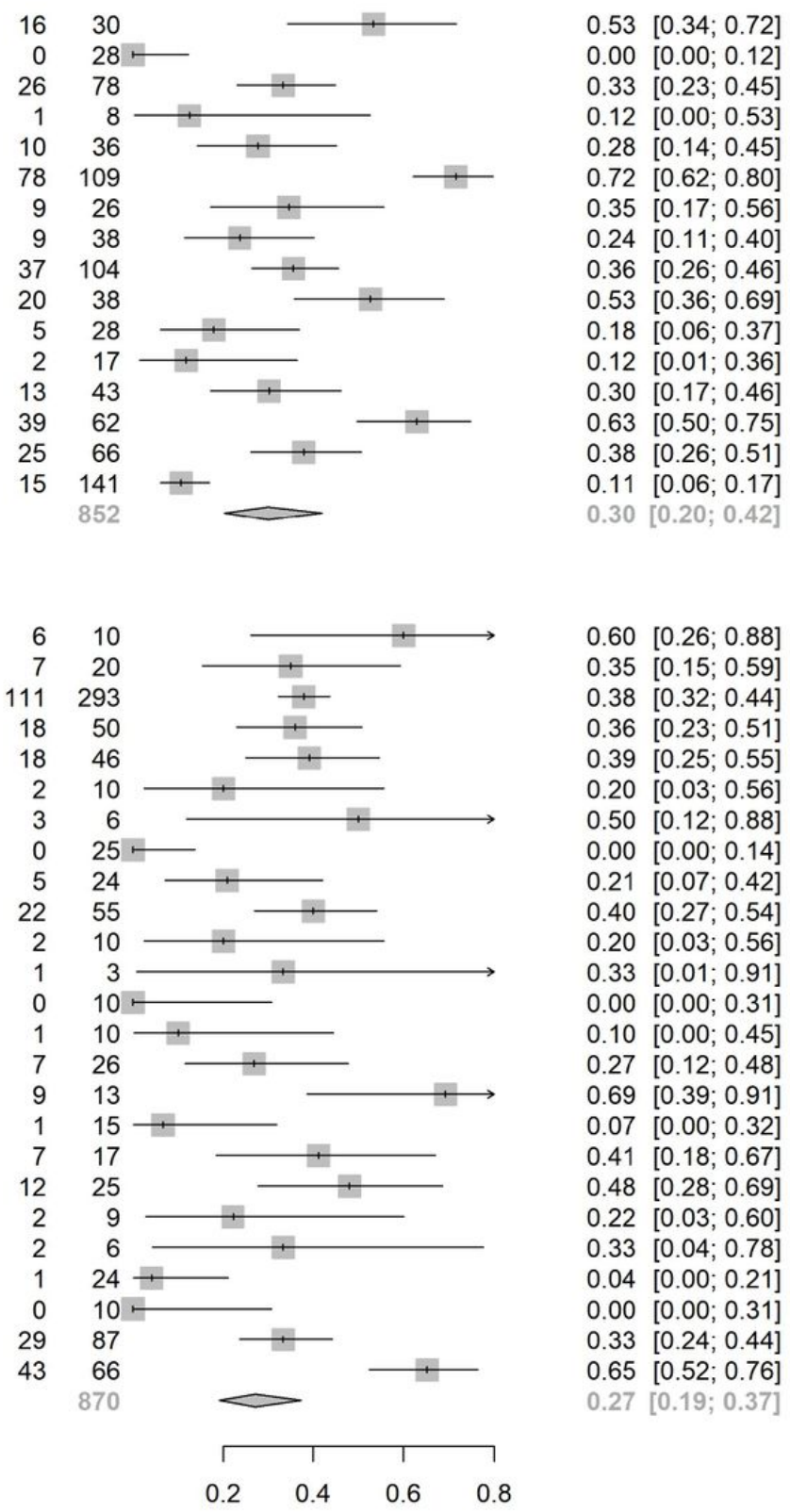

Figure 2

Association between awake prone positioning and intubation, in each report, and overall 
Subgroups

Duration

$<4$ hours

4 hours and more

178

165

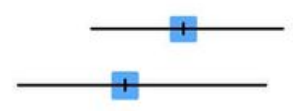

604

453

158

73

50

77

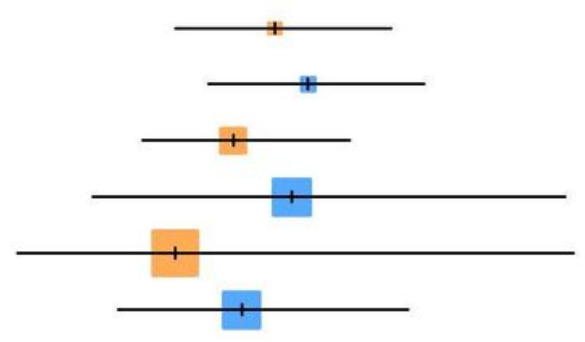

0.34 [0.22;0.48]

$0.38[0.26 ; 0.52]$

$0.29[0.18 ; 0.43]$

$0.36[0.12 ; 0.69]$

$0.22[0.03 ; 0.70]$

$0.30[0.15 ; 0.50]$

$0.35[0.23 ; 0.50]$

$0.39[0.35 ; 0.44]$

$0.08[0.03 ; 0.19]$

$0.23[0.13 ; 0.38]$
$0.35[0.24 ; 0.47]$

$0.28[0.15 ; 0.45]$
P/F above 150, PP 192

441

479

$P / F<150$, no $P P$

$P / F<150, P P$
Proportions

$95 \%-\mathrm{Cl}$

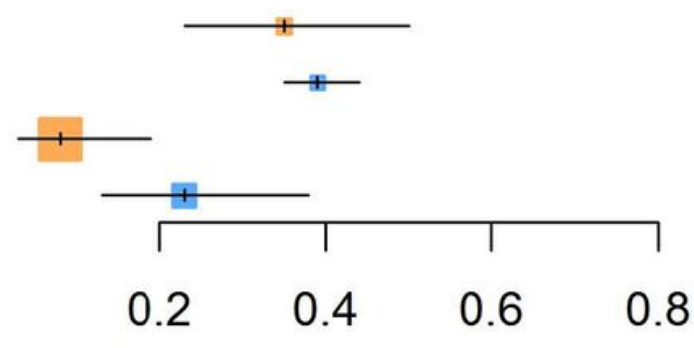

$\begin{array}{llll}0.2 & 0.4 & 0.6 & 0.8\end{array}$

\section{Figure 3}

Association between awake prone positioning and intubation, within subgroups defined by the du-ration of proning, the type of respiratory support device, and the $\mathrm{PaO} 2 / \mathrm{FiO} 2$ ratio at enrolment. 
No prone positioning

Burns et al, 2020

Duca et al, 2020

Geng et al, 2020

He et al, 2020

Hernandez-Romieu et al, 2020

Knights et al, 2020

Oranger et al, 2020

Pagano et al, 2020

Patel et al, 2020

Sivaloganathan et al, 2020

Vianello et al, 2020

Xia et al, 2020

Unpublished data from Duan et al (China)

Unpublished data from UChicago Medicine

Random effects model

Heterogeneity: $I^{2}=92 \%, \tau^{2}=1.3768, p<0.01$

Prone positioning

Burton-Papp et al, 2020

Calligaro et al, 2020

Coppo et al, 2020

Damarla et al, 2020

Dong et al, 2020 (Preprint)

Ferrando et al, 2020

Golestani-Eraghi et al, 2020

Moghadam et al, 2020

$\mathrm{Ng}$ et al, 2020

Retucci et al., 2020

Ripoll-Gallardo et al, 2020

Sartini et al, 2020

Solverson et al, 2020

Thompson et al, 2020

Villarreal-Fernandez et al, 2020

Winearls et al, 2020

Xu et al, 2020

Zang et al, 2020

Unpublished data from the Irish awake proning group Unpublished data from Unity Point Health

Random effects model

Heterogeneity: $I^{2}=87 \%, \tau^{2}=1.4291, p<0.01$

Heterogeneity: $I^{2}=91 \%, \tau^{2}=1.5466, p<0.01$

Test for subgroup differences: $\chi_{1}^{2}=2.78, \mathrm{df}=1(p=0.10)$

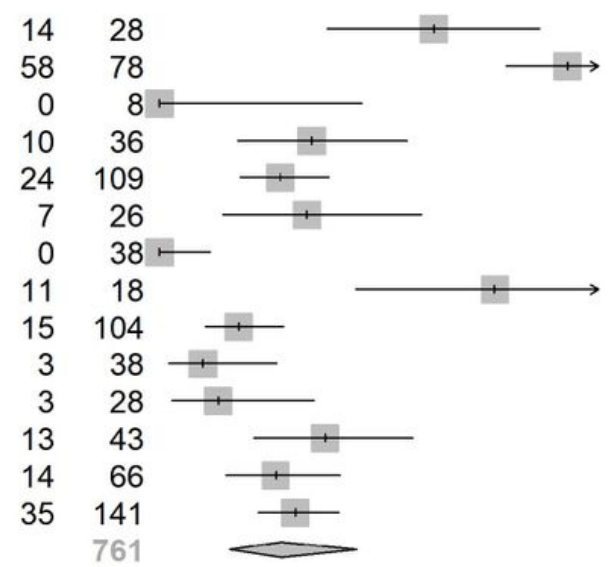

$0.50[0.31 ; 0.69]$

$0.74[0.63 ; 0.84]$

$0.00[0.00 ; 0.37]$

$0.28[0.14 ; 0.45]$

$0.22[0.15 ; 0.31]$

$0.27[0.12 ; 0.48]$

$0.00[0.00 ; 0.09]$

$0.61[0.36 ; 0.83]$

$0.14[0.08 ; 0.23]$

$0.08 \quad[0.02 ; 0.21]$

$0.11[0.02 ; 0.28]$

$0.30[0.17 ; 0.46]$

$0.21[0.12 ; 0.33]$

$0.25[0.18 ; 0.33]$

$0.22[0.13 ; 0.36]$

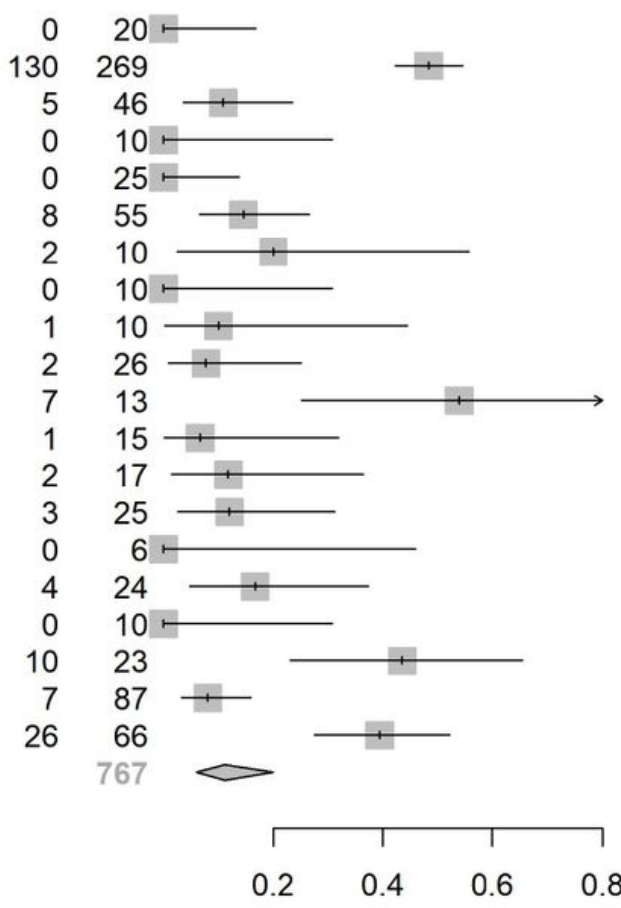

$0.00 \quad[0.00 ; 0.17]$

$0.48[0.42 ; 0.54]$

$0.11[0.04 ; 0.24]$

$0.00[0.00 ; 0.31]$

$0.00[0.00 ; 0.14]$

$0.15[0.06 ; 0.27]$

$0.20[0.03 ; 0.56]$

$0.00[0.00 ; 0.31]$

$0.10[0.00 ; 0.45]$

$0.08[0.01 ; 0.25]$

$0.54[0.25 ; 0.81]$

$0.07[0.00 ; 0.32]$

$0.12[0.01 ; 0.36]$

$0.12[0.03 ; 0.31]$

$0.00[0.00 ; 0.46]$

$0.17[0.05 ; 0.37]$

$0.43[0.23 ; 0.66]$

$0.08[0.03 ; 0.16]$

$0.39[0.28 ; 0.52]$

$0.11[0.06 ; 0.20]$
$0.00 \quad[0.00 ; 0.31]$

\section{Figure 4}

Association between awake prone positioning and mortality, in each report, and overall. 
Subgroups

Duration

$<4$ hours

4 hours and more

Method

HFNC, no PP

HFNC, PP

CPAP, no PP

CPAP, PP

NIV, no PP

NIV, PP n

Proportions
$0.20[0.08 ; 0.41]$

0.12 [0.08; 0.19]

$0.23[0.17 ; 0.29]$

$0.25[0.12 ; 0.45]$

$0.44[0.20 ; 0.70]$

$0.22[0.06 ; 0.56]$

$0.28[0.06 ; 0.71]$

$0.12[0.06 ; 0.22]$

$0.28[0.11 ; 0.55]$

$0.18[0.08 ; 0.34]$

$0.23[0.01 ; 0.29]$

$0.09[0.06 ; 0.15]$

P/F above 150, no PP 58

P/F above 150, PP

186

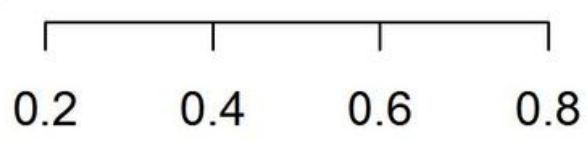

Figure 5

Association between awake prone positioning and mortality, within subgroups defined by the duration of proning, the type of respiratory support device, and the $\mathrm{PaO} 2 / \mathrm{FiO}_{2}$ at enrolment.

\section{Supplementary Files}

This is a list of supplementary files associated with this preprint. Click to download.

- Table1.xlsx

- OnlinesupplementTableE1E2andFiguresE1E26.pdf 